Authors have nothing to disclose with regard to commercial support.

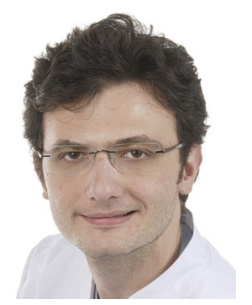

\section{OUTCOME BENEFITS AFTER CORONARY ARTERY BYPASS GRAFT IN LOW- VERSUS HIGH-VOLUME CENTERS: IS IT ALL ABOUT THE SURGERY?}

\section{To the Editor:}

We have read with great interest the recently published study by Kim and colleagues. ${ }^{1}$ They show that in-hospital adverse events and all-cause mortality after coronary artery bypass graft (CABG) surgery have decreased over the years. In a multivariate logistic regression analysis, however, low hospital volume of CABG surgery was an independent predictor of in-hospital all-cause mortality (adjusted odds ratio, 1.39; 95\% confidence interval, 1.24-1.56; $P<.001$ ), as compared with the outcome in high-volume centers. A detailed examination of the postoperative complications among elective referrals, respiratory failure $(P<.001)$, postoperative bleeding $(P<.001)$, and acute renal failure $(P=.02)$ showed significantly higher rates in low-volume centers than in high-volume centers. As a consequence, the authors advocate more intensive assessments of the quality of surgical care in low-volume institutions.

In our view, this claim focuses on only part of the problem and its remedy. Besides the importance of the surgeon and his performance, other aspects such as preoperative assessment and scheduling, intraoperative management by the theatre team (eg, surgeon, nurses, perfusionist, anesthesiologist), and especially a sound postoperative treatment in the intensive care unit (ICU) are also important. Moreover, early recognition, prevention strategies, and troubleshooting capabilities in postoperative care most likely complete the bundle of high-class health care. To focus only on the experience of the surgeon in the low-volume centers might be an unjustified simplification of a multidisciplinary complex procedure.

\footnotetext{
The Editor welcomes submissions for possible publication in the Letters to the Editor section that consist of commentary on an article published in the Journal or other relevant issues. Authors should: • Include no more than 500 words of text, three authors, and five references. $\bullet$ Type with double-spacing. $\bullet$ See http://jtcs.ctsnetjournals.org/ misc/ifora.shtml for detailed submission instructions. • Submit the letter electronically via jtcvs.editorialmanager.com. Letters commenting on an article published in the JTCVS will be considered if they are received within 6 weeks of the time the article was published. Authors of the article being commented on will be given an opportunity of offer a timely response ( 2 weeks) to the letter. Authors of letters will be notified that the letter has been received. Unpublished letters cannot be returned.
}

Indeed, this notion is undermined by a multitude of publications. In a systematic review and meta-analysis, for example, Nguyen and colleagues ${ }^{2}$ showed that critically ill patients after surgery for cardiovascular conditions benefit from care in high-volume centers. Sasabuchi and colleagues $^{3}$ reported that a higher hospital volume of ICU patients and a higher ICU-to-hospital bed ratio were independently associated with lower mortality. In another study dealing only with a cardiac surgery population, Kogan and colleagues ${ }^{4}$ found a significant reduction of morbidity and mortality after implementation of quality-improving tools such as multidisciplinary ICU rounds, a glucose control protocol, sepsis treatment protocols, a ventilator bundle, early nutritional support, and so forth. Failure to properly react to problems was addressed in a retrospective study that included 119,434 patients who underwent high-risk cardiac surgery. The higher the volume of patients passing through the hospital, the less frequently failure to rescue occurred; a finding that directly translated into improved survival in high-volume centers despite the complication rates not correlating with the volume of patients.

Thus, results after CABG must not solely be defined by the preoperative risk factors, the surgeon's performance, or the volume of the institutions; the ICU volume of a given center and the standard of care provided throughout the perioperative flow by the multiprofessional team must also be considered.

$$
\begin{array}{r}
\text { Angelo M. Dell'Aquila, } M D^{a} \\
\text { Ingeborg van den Heuvel, } M D^{b} \\
\text { Bjoern Ellger, } M D^{c} \\
\text { Departments of }{ }^{a} \text { Cardiothoracic Surgery and } \\
{ }^{c} \text { Anesthesiology } \\
\text { Intensive Care Medicine and Pain Therapy } \\
\text { University Hospital Muenster and } \\
{ }^{b} \text { Department of General Pediatrics } \\
\text { University Children's Hospital Muenster } \\
\text { Muenster, Germany }
\end{array}
$$

\section{References}

1. Kim LK, Looser P, Swaminathan RV, Minutello RM, Wong SC, Girardi L, et al Outcomes in patients undergoing coronary artery bypass graft surgery in the United States based on hospital volume, 2007 to 2011. J Thorac Cardiovasc Surg. 2016;151:1686-92.

2. Nguyen YL, Wallace DJ, Yordanov Y, Trinquart L, Blomkvist J, Angus DC, et al. The volume-outcome relationship in critical care: a systematic review and metaanalysis. Chest. 2015;148:79-92.

3. Sasabuchi Y, Yasunaga H, Matsui H, Lefor AK, Horiguchi H, Fushimi K, et al. The volume-outcome relationship in critically ill patients in relation to the ICU-tohospital bed ratio. Crit Care Med. 2015;43:1239-45.

4. Kogan A, Preisman S, Berkenstadt H, Segal E, Kassif Y, Sternik L, et al. Evaluation of the impact of a quality improvement program and intensivist-directed ICU team on mortality after cardiac surgery. J Cardiothorac Vasc Anesth. 2013;27: 1194-200.

5. Gonzalez AA, Dimick JB, Birkmeyer JD, Ghaferi AA. Understanding the volumeoutcome effect in cardiovascular surgery: the role of failure to rescue. JAMA Surg. 2014;149:119-23.

http://dx.doi.org/10.1016/j.jtcvs.2016.05.054 